\title{
Section B
}

\section{Theoretical tools}




\title{
Morphological statistics of the cosmic web
}

\author{
Sergei F. Shandarin \\ Department of Physics and Astronomy, University of Kansas, KS 66045, USA \\ email: sergei@ku.edu
}

\begin{abstract}
We report the first systematic study of the supercluster-void network in the $\Lambda$ CDM concordance cosmology treating voids and superclusters on an equal footing. We study the dark matter density field in real space smoothed with the $L_{s}=5 h^{-1} \mathrm{Mpc}$ Gaussian window. Superclusters and voids are defined as individual members of over-dense and under-dense excursion sets respectively. We determine the morphological properties of the cosmic web at a large number of dark matter density levels by computing Minkowski functionals for every supercluster and void. At the adopted smoothing scale individual superclusters totally occupy no more than about $5 \%$ of the total volume and contain no more than $20 \%$ of mass if the largest supercluster is excluded. Likewise, individual voids totally occupy no more than $14 \%$ of volume and contain no more than $4 \%$ of mass if the largest void is excluded. The genus of individual superclusters can be $\sim 5$ while the genus of individual voids reaches $\sim 55$, implying significant amount of substructure in superclusters and especially in voids. Large voids are typically distinctly nonspherical.
\end{abstract}

\section{Introduction}

Redshift galaxy catalogues reveal a universe permeated by an interpenetrating network of superclusters and voids. It therefore becomes important to understand and quantify the geometrical and topological properties of large scale structure in an $\Lambda$ CDM cosmology in a deep and integrated manner. The main aim of this talk is to study the superclustervoid network in $\Lambda$ CDM cosmology with emphasis on the sizes, shapes and topologies of individual superclusters and voids. Concretely, we study the geometry and topology of isodensity surfaces $\delta(\mathbf{x}) \equiv \delta \rho(\mathbf{x}) / \bar{\rho}=$ const. At a given threshold $\delta_{t h}$ regions having higher then threshold density $\left(\delta>\delta_{t h}\right)$ will be called "superclusters", while regions with $\delta<\delta_{t h}$ will be called "voids". We employ an elaborate surface modeling scheme, SURFGEN (short for 'surface generator'), that allows us to determine the geometry, morphology and topology of excursion sets defined on a density field in a very comprehensive manner (Sheth et al. 2003). Working with the density field also permits us to determine the morphological properties of the full excursion set describing the supercluster-void network. More detailed information is then gleaned at one particular threshold (usually associated with percolation) at which shapes and sizes of individual superclusters and voids yield rich information about properties of the cosmic web to which we belong.

We use dark matter distributions in a flat model with $\Omega_{0}=0.3, \Omega_{\Lambda}=0.7, h=0.7$ $(\Lambda \mathrm{CDM})$. The initial spectrum was taken with the shape parameter $\Gamma=0.21$. The amplitude $\left(\sigma_{8}=0.9\right)$ of the power spectrum in the model is set so as to reproduce the observed abundance of rich galaxy clusters at the present epoch (see Jenkins et al. 1998 for details). The study of mock galaxy distributions was done by Sheth (2003).

SURFGEN operates on three-dimensional pixelized maps. Therefore we first generate the density field from the distribution of dark matter particles. This process was described in detail in Sheth et al. (2003); here we present a brief summary. The data consist of $256^{3}$ particles in a box of size $239.5 h^{-1} \mathrm{Mpc}$. We fit a $128^{3}$ grid to the box. We follow the smoothing technique used by Springel et al. (1998) which they adopted 
for their preliminary topological analysis of the Virgo simulations. We study the field smoothed with $L_{s}=5 h^{-1} \mathrm{Mpc}$ which is a fiducial smoothing scale in many studies of both density fields in N-body simulations and galaxy fields from redshift surveys; see for example, Grogin \& Geller (2000). The Gaussian kernel for smoothing that we adopt here is $W(r)=\pi^{-3 / 2} L_{s}^{-3} \exp \left(-r^{2} / L_{s}^{2}\right)$. Since the kernel is isotropic and uniform, it is likely to diminish the true extent of anisotropy in filaments, pancakes and voids. This effect could be minimized by considering adaptive kernels or smoothing techniques based on the wavelet transform. An even more ambitious approach is to reconstruct density fields using Delaunay tesselations using a technique reported by van de Weygaert (2002).

Four Minkowski functionals (MFs) are effective non-parametric descriptors of the morphological properties of surfaces in three dimensions Mecke et al. (1994). They are: Volume $V$ enclosed by the surface, $S$; Area $A$ of the surface; Integrated mean curvature $C$ of the surface $C=\frac{1}{2} \oint_{S}\left(\frac{1}{R_{1}}+\frac{1}{R_{2}}\right) d a$, where $R_{1}$ and $R_{2}$ are the principal radii of curvature at a given point on the surface; and finally, the Euler characteristic $\chi=\frac{1}{2 \pi} \oint_{S}\left(\frac{1}{R_{1} R_{2}}\right) d a$. The genus is uniquely related to the Euler characteristic $G=1-\chi / 2$ and thus carries exactly the same information. We measure the above parameters for every region in both over-dense and under-dense excursion sets at 99 density thresholds equispaced in the filling factor from $F F=0.01$ to $F F=0.99$ where $F F$ is the fraction of the total volume occupied by the excursion set. As demonstrated in Sahni et al. (1998) and Sathyaprakash et al. (1998), particular ratios of the MFs called "Shapefinders" provide us with a set of non-parametric measures of sizes and shapes of objects. Therefore, in addition to determining MFs we shall also derive three quantities having the dimension of length that can be associated with three characteristic sizes: $T$ (Thickness), $B$ (Breadth) and $L$ (Length) defined as follows: $T=3 V / A, B=A / C, \quad L=C / 4 \pi$. The three Shapefinders describing an individual region bounded by one or several isolated surfaces of constant density have dimension of length and provide us with an estimate of the region extensions. The choice of the coefficients in the above equations results in a sphere having all three sizes equal to its radius $T=B=L=R$. It is worth noting that $T, B$ and $L$ are only the estimates of three basic sizes (semi-axes) of an object, which work quite well on objects such as a triaxial ellipsoid and a torus. An indicator of 'shape' is provided by a pair of dimensionless Shapefinder statistics $P=(B-T) /(B+T) ; \quad F=(L-B) /(L+B)$, where $P$ and $F$ are measures of Planarity and Filamentarity respectively $(P, F \leqslant 1)$. A sphere has $P=F=0$, an ideal filament has $P=0, F=1$, while $P=1, F=0$ for an ideal pancake.

\section{Results}

Understanding percolation is essential for understanding the morphology of the supercluster - void network. Percolation is important because the properties of superclusters and voids radically change at the percolation transitions (see Fig. 1). At relatively high thresholds $\delta_{C}>1.8$, corresponding to small filling factors $F F_{C}<0.07$, the largest supercluster has insignificant volume and mass compared to the total volume or mass contained in the over-dense excursion set, $\delta>\delta_{\mathrm{TH}}$. During the percolation transition at $F F_{C} \approx 0.07$, corresponding to $\delta_{C} \approx 1.8$, both volume and mass in the largest supercluster rapidly grow, overtaking the volume and mass in the entire excursion set, and completely dominating the entire sample from this point onwards. The largest void behaves in a qualitatively similar manner if plotted versus $F F_{V}$. At $F F_{V}<0.22, \delta_{V}<-0.5$ its volume is small compared to the volume of the under-dense excursion set, $\delta<\delta_{\mathrm{TH}}$, but at the percolation transition $F F_{V} \approx 0.22, \delta_{V} \approx-0.5$, it takes over and from then on it remains 

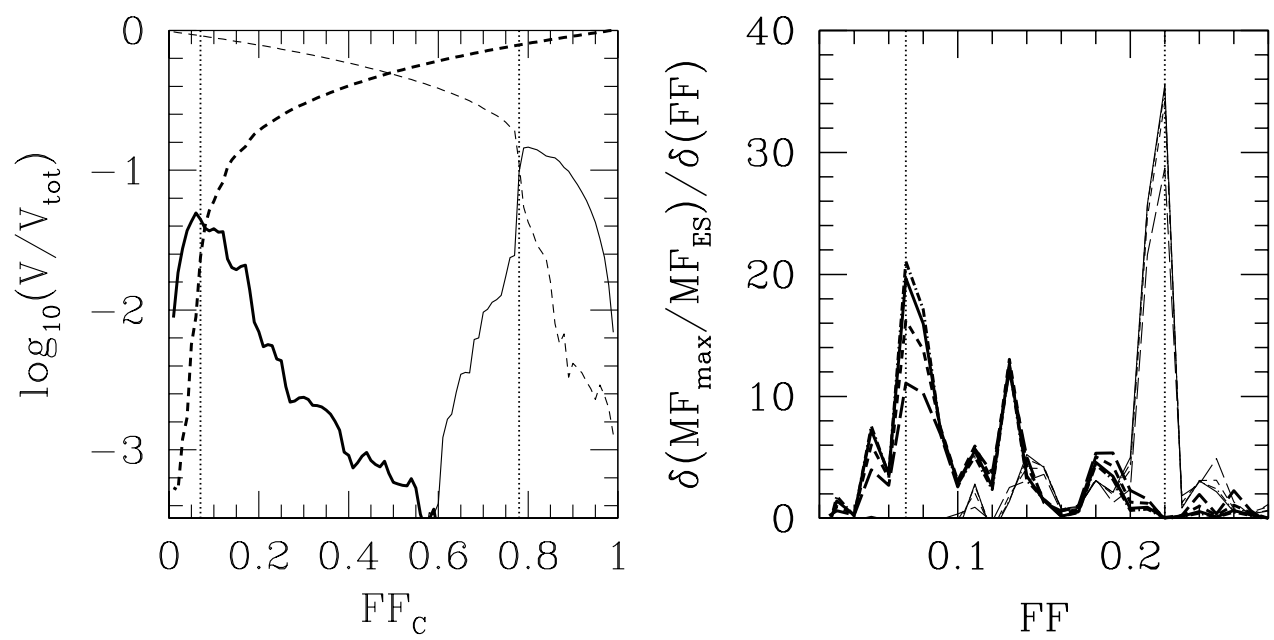

Figure 1. Left: the fractions of the total volume occupied by the largest supercluster (thick dashed line), all superclusters but the largest one (thick solid line), largest void (thin dashed line), and all voids but the largest one (thin solid line) are shown for the density field in the $\Lambda \mathrm{CDM}$ model smoothed with $L_{s}=5 h^{-1} \mathrm{Mpc}$ as a function of the filling factor, $F F_{C}$. Right: the estimates of the percolation thresholds. Thick lines show the rate of growth $\delta m^{(i)} / \delta(F F)$ for four estimators (Shandarin et al. 2003) for superclusters as a function of $F F_{C}$. All four curves consistently peak at $F F_{C}=0.07$. Thin lines show similar quantities for voids with a distinct peak at $F F_{C}=0.22$. Vertical dotted lines mark the percolation thresholds.

the dominant structure in the under-dense excursion set. Since $F F_{C} \equiv 1-F F_{V}$ the void percolation transition takes place at $F F_{C} \approx 0.78$ as shown in Fig. 1 (left panel). Two obvious conclusions can be drawn from the above discussion. First, at percolation the object having the largest volume becomes very different from all the remaining objects, therefore it must be studied separately. Second, individual objects - both superclusters and voids - must be studied in the corresponding phase before percolation occurs in the corresponding phase. Both superclusters and voids reach their largest sizes, volumes and masses just before percolation sets in. The percolation thresholds can be accurately measured from the peaks in the rate of growth of the MFs of the largest supercluster or void as a function of FF (Fig. 1 right panel).

It is interesting to compare the percolation and genus statistics. Both were suggested as tests for the connectedness of the large-scale structure. First, Zel'dovich and Shandarin (Zel'dovich 1982, Shandarin 1983, Shandarin \& Zel'dovich 1983) raised the question of topology of large-scale structure and suggested percolation statistic as a discriminator between models. The percolation test was first applied to a redshift catalog compiled by J. Huchra and Rood (Zel'dovich et al. 1982; Einasto et al. 1984) who found that the connectedness in this catalog was significantly stronger than for a Poisson distribution. In contrast, a non-dynamical model having approximately correct correlation functions up to the fourth order (Soneira \& Peebles 1978) showed significantly weaker connectedness than in the observed catalog. Thus, percolation was able to detect connectedness in the galaxy distribution. It was also demonstrated that three lowest order correlation functions (two-, three- and four-point functions) are not sufficient to detect the connectedness in the galaxy distribution. Figure 2 illustrates the difference between the global genus (Fig. 2 left panel) and percolation statistic (Fig. 1 left panel and Fig. 2 right panel): at the percolation threshold many properties of the excursion set significantly change while the global genus curve remain smooth and featureless. 

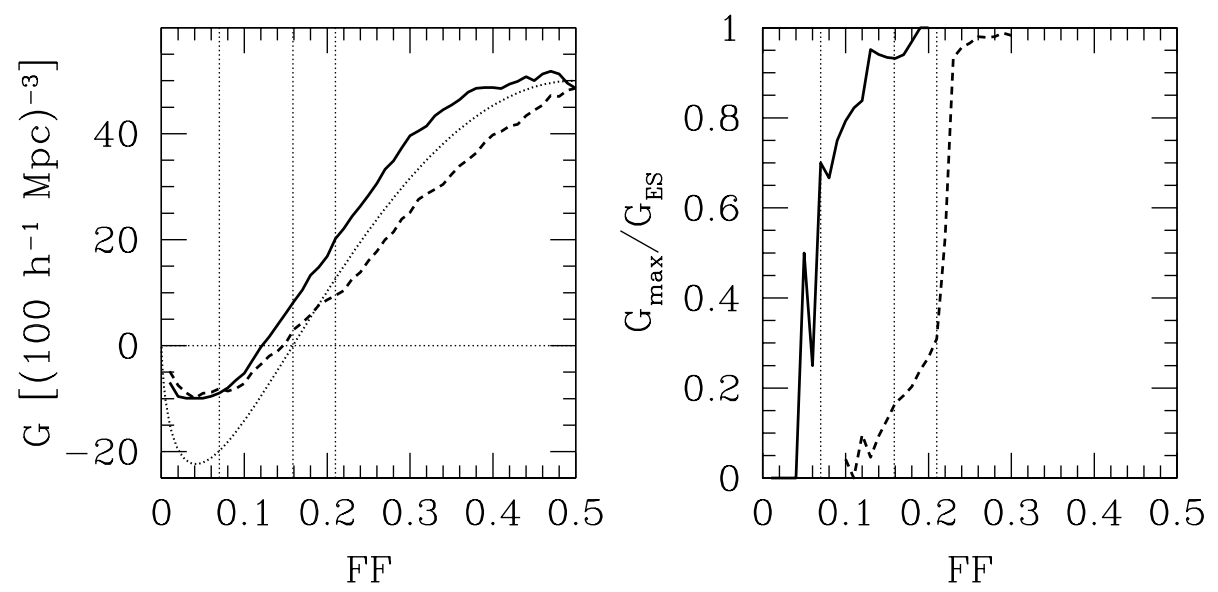

Figure 2. Left: the global genus is shown as a function of the filling factor for the density field smoothed with $L_{s}=5 h^{-1}$ Mpc. The half of the curve corresponding to high density thresholds is plotted as a function of $F F_{C}$ (solid line) while the other half corresponding to low density thresholds is plotted as a function of $F F_{V}$ (dashed line). For comparison, the dotted line shows the Gaussian genus curve having the same amplitude. Right: the percolation transitions in the same density field as indicated by the genus of the largest supercluster (solid line) and largest void (dashed line). The vertical dotted lines mark two percolation thresholds in the $\Lambda$ CDM $(F F \approx 0.07$ and $F F \approx 0.22)$ and Gaussian field $(F F \approx 0.16)$ in both panels.
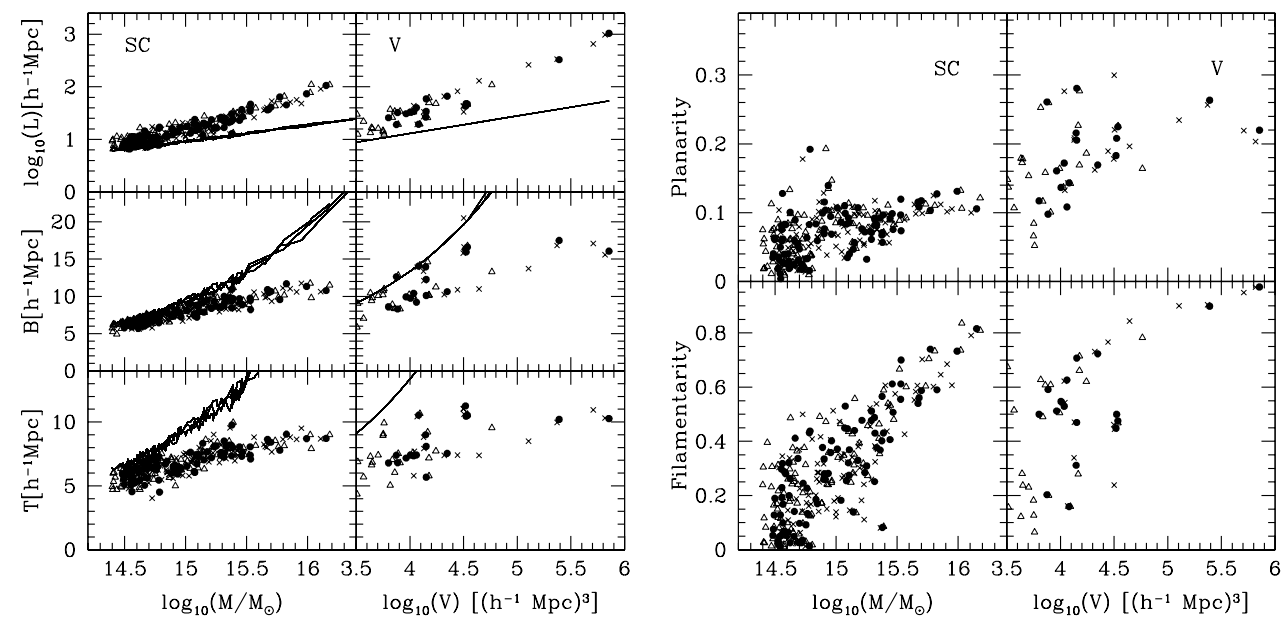

Figure 3. Left: length, breadth, and thickness versus mass for superclusters and versus volume for voids. Solid circles show the relation at percolation thresholds: $F F_{C}=0.07$ for superclusters and $F F_{V}=0.22$ for voids. Crosses show the parameters before percolation $\left(F F_{C}=0.06\right.$ for superclusters and $F F_{V}=0.21$ for voids) and empty triangles after percolation $\left(F F_{C}=0.08\right.$ for superclusters and $F F_{V}=0.23$ for voids). Solid lines show the radius of the sphere having the same volume as the supercluster or void. Right: planarity and filamentarity vs mass (for superclusters) and vs volume (for voids) at percolation. Notations are as in the left panel.

Three characteristic sizes and shapes of superclusters and voids can be estimated from the MFs. It is surprising that the thickness of superclusters depends on the threshold quite weakly; it is within $4-6 h^{-1} \mathrm{Mpc}$ interval for a range of thresholds between $0<\delta<6$. This may indicate that the actual thickness of superclusters is significantly smaller and the 

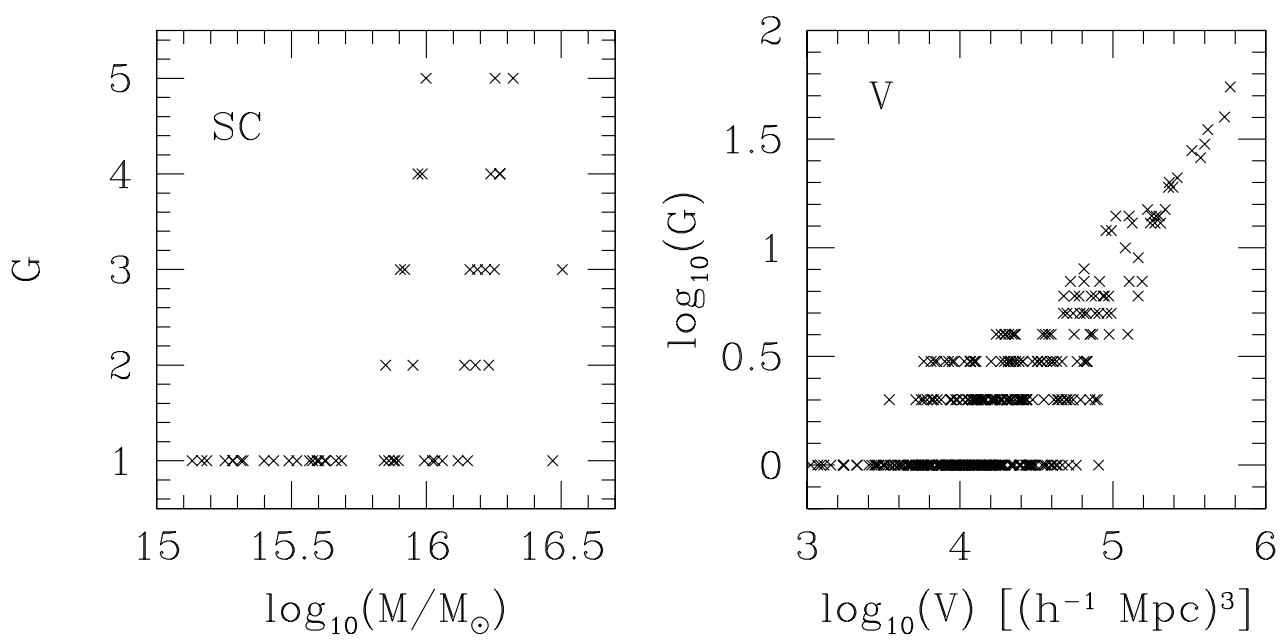

Figure 4. Left: genus-mass relation for superclusters. Right: genus-volume relation for voids. Every isolated supercluster and void having genus greater than zero at all density thresholds is shown.

measured values reflect the width of the smoothing window. The breadth of superclusters is not much greater than the thickness and it is likely that it is also affected by the width of the filtering window. Voids are a little fatter than superclusters and their thickness reaches about $9 h^{-1} \mathrm{Mpc}$ at the percolation threshold. Voids are also wider and longer than superclusters. Recalling that the size parameters are normalized to the radius of the sphere rather than to the diameter, we conclude that the longest $25 \%$ of superclusters are longer than about $50 h^{-1} \mathrm{Mpc}$ and $25 \%$ of voids are longer than about $60 h^{-1} \mathrm{Mpc}$.

The correlation of the sizes $(L, B, T)$ and shape parameters $(P, F)$ of superclusters and voids with the masses (superclusters) and volumes (voids) are shown in Fig. 3. It was expected that all three sizes of the superclusters may correlate with their masses and the sizes of the voids correlate with their volumes. It is perhaps also not surprising that the filamentarity of superclusters increases with the mass. However, the strong correlation of the filamentarity $(F)$ of voids with their volumes is quite unexpected and may require reconsidering some theoretical models of the void evolution. Figure 4 (left panel) demonstrates that the planarity of superclusters is quite small, indicating that pancakes are not typical structures in the $\Lambda$ CDM cosmology. In addition, we have found a very strong indication of complex geometry and nontrivial topology in the largest superclusters (Fig. 4 left panel) and especially in voids (Fig. 4 right panel).

\section{Summary}

Individual superclusters totally occupy no more than about $5 \%$ of the total volume and comprise no more than $20 \%$ of mass if the largest (i.e. percolating) supercluster is excluded. The maximum of the total volume and mass comprised by all superclusters, except the largest one, is reached approximately at the percolation threshold: $\delta \approx 1.8$ corresponding to $F F_{C} \approx 0.07$. Individual voids totally occupy no more than $14 \%$ of volume and contain no more than $4 \%$ of mass if the largest void is excluded. The maximum of the total volume and mass comprised by all voids except the largest one is reached at about the void percolation threshold: $\delta \approx-0.5$ corresponding to $F F_{V} \approx 0.22$. Between these two percolation thresholds all superclusters and voids except the largest ones take 
up no more than about $10 \%$ of volume and mass. Both the largest supercluster and void span throughout the whole space and have a very large genus. Therefore neither of them has well defined sizes, volumes, masses or easily defined shapes.

The sizes of voids are significantly larger than those of superclusters even in the density field smoothed with $L_{s}=5 h^{-1} \mathrm{Mpc}$. The length of a quarter of the most massive superclusters exceeds $50 h^{-1} \mathrm{Mpc}$. The most voluminous voids are even longer: $25 \%$ of them are longer than $60 h^{-1} \mathrm{Mpc}$. The longest non-percolating supercluster is as long as $100 h^{-1} \mathrm{Mpc}$ and the longest non-percolating void is as long as $200 h^{-1} \mathrm{Mpc}$. Both are comparable to the size of the box $\left(239.5 h^{-1} \mathrm{Mpc}\right)$ and therefore may be affected by the boundaries.

The genus value of individual superclusters can be $\sim 5$ while the genus of individual voids can reach $\sim 55$ (Fig. 4). This implies significant amount of substructure in superclusters and especially in voids. This is in a general agreement with other studies of voids (Kofman et al. 1992; Peebles 2001; Gottlöber et al. 2003).

One of our main results is that voids, as defined through the density field, can be distinctly non-spherical. Whether this result carries over to voids in galaxy surveys will depend upon the nature of the baryon-dark matter biasing and also on whether the density field is sampled in real or in redshift space.

The percolation thresholds as well as some other parameters depend on the smoothing scale and for smaller smoothing scales or adaptive filtering windows the supercluster percolation threshold must decrease $\left(F F_{C}^{\text {perc. }}<0.07\right)$ and the void percolation threshold increase $\left(F F_{V}^{\text {perc. }}>0.22\right)$.

\section{Acknowledgements}

I am thankful to Antonaldo Diaferio for a careful reading of the manuscript and useful critical comments.

\section{References}

Cole, S., Hatton, S., Weinberg, D.H., Frenk, C.S., 1998, MNRAS, 945

Einasto, J., Klypin, A.A., Saar, E., Shandarin, S.F., 1984, MNRAS, 206, 529

Gott, J.R., Melott, A.L. \& Dickinson, M., 1986, ApJ, 306, 341

Gottlöber, S., Lokas, E.L., Klypin, A. Hoffman, Y., 2003, MNRAS, 344, 715

Jenkins, A.R. et al. (for the Virgo Constortium), 1998, ApJ, 499, 20

Kofman, L.A., Pogosyan, D., Shandarin, S.F. and Melott, A., 1992, ApJ, 393, 450.

Mecke, K.R., Buchert, T. \& Wagner, H., 1994, A\&A, 288, 697

Peebles, P.J.E., 2001, ApJ, 557,495

Sahni, V., Sathyaprakash, B.S. \& Shandarin, S.F., 1998a, ApJL, 495, L5

Sathyaprakash, B.S., Sahni, V. \& Shandarin, S.F., 1998b, ApJ, 508, 551

Sheth, J.V. 2003, astro-ph/0310755

Sheth, J.V., Sahni, V., Shandarin, S.F. \& Sathyaprakash, B. 2003, MNRAS, 343, 22

Shandarin, S.F. 1983, Sov. Astron. Lett., 9, 104

Shandarin, S.F. \& Zeldovich, Ya.B., 1983, Comments Astrophys. 10, 33

Shandarin, S.F. \& Zeldovich, Ya.B., 1989, Rev. Mod. Phys.,61,185

Shandarin, S.F. Sheth, J., \& Sahni, V. 2003, astro-ph/0312110

Soneira, R.M. \& Peebles, P.J.E. 1978, Astron. J., 83, 845

Springel, V. et al. (for the Virgo Consortium), 1998, MNRAS, 298, 1169

van de Weygaert, R., 2002, astro-ph/0206427

Zel'dovich, Ya.B. 1982, Sov. Astron. Lett., 8, 102

Zeldovich, Ya.B., Einasto, J. and Shandarin, S.F., 1982, Nature, 300, 407 